\title{
26 Research Square \\ Study on the Changes of Structural Covariance Network in Post-traumatic Stress Disorder
}

\section{Tongtong Xu}

Nanjing Normal University School of Psychology

\section{Feng Chen}

Hainan General Hospital

\section{Li Zhang}

Second Xiangya Hospital Mental Health Institute

\section{Yingliang Dai}

Nanjing Normal University School of Psychology

Jun Ke

First Affiliated Hospital of Soochow University

Rongfeng Qi

Medical School of Nanjing University: Nanjing University Medical School

Yuan Zhong ( $\sim$ zhongyuan@njnu.edu.cn )

Nanjing Normal University School of Psychology https://orcid.org/0000-0002-4237-3206

Guangming Lu

Medical School of Nanjing University: Nanjing University Medical School

\section{Research Article}

Keywords: PTSD, Cortical thickness, Structural covariance network, Graph theory, Small world

Posted Date: August 10th, 2021

DOI: https://doi.org/10.21203/rs.3.rs-779634/v1

License: (c) (i) This work is licensed under a Creative Commons Attribution 4.0 International License.

Read Full License 


\section{Abstract}

It has been widely discussed that the topological characteristics of the functional brain network in posttraumatic stress disorder (PTSD) demonstrates impairments, but the topology of structural covariance network has rarely been studied. Based on graph theoretical approaches, we compared the topological organization of structural covariance networks among PTSD, trauma-exposed control (TEC) group and healthy control $(\mathrm{HC})$ group by constructing covariance networks of cortical thickness. Structural magnetic resonance imaging (sMRI) scans and clinical scales were performed in 27 PTSD, 33 TEC and 29 HC subjects. Based on the cortical thickness correlations, morphological network was constructed and analyzed for each group. Pearson correlation coefficients were computed to explore the relationship of brain network measurements with clinical scales. PTSD and HC subjects did not differ at the global level. Significantly increased clustering coefficient $(p=.014)$ and local efficiency $(p=.031)$ were observed in PTSD group compared with TEC subjects. At the nodal level, PTSD showed extensive increased nodal centralities in comparison to the other two groups ( $p<.05$, uncorrected for false-discovery rate). The structural brain network topological characteristics found in this study suggest that PTSD manifests differently compared to TEC group from pathophysiological perspective. Greater network separation and increased nodal centrality in PTSD patients may be concerning emotional disorders.

\section{Introduction}

Post-traumatic stress disorder (PTSD) is a chronic psychiatric disorder (Francesmonneris et al., 2013). An epidemiological survey portrays that the lifetime prevalence of PTSD in China is 4\% (Huang et al., 2019). Network-based analysis may provide us with a more comprehensive understanding of the pathogenesis of PTSD.

Graph theory is used for analyzing the topological characteristics of the brain network (Bullmore \& Sporns, 2009; Rubinov \& Sporns, 2010). According to this method, previous studies using different neuroimaging methods have revealed abnormalities in the brain networks characteristics in PTSD (Niu, Du, et al., 2018; Proessl et al., 2020; Suo et al., 2019; Zhu et al., 2019). As a new method for constructing large-scale brain networks, structural covariance network (SCN), which reflects the correlation of morphological indicators between brain regions (Tijms et al., 2012; Zhang J. Chen \& Evans, 2008), has been applied in many neurological diseases research (Cai et al., 2021; Guo et al., 2018).

A healthy brain can balance information integration and specialized processing, that is, it has a smallworld topology (He et al., 2007; Sporns \& Zwi, 2004). Some studies based on SCN have reported reduced small-world property in PTSD. Qi and colleagues studied cortical thickness covariance networks in newly onset PTSD patients and found that compared with healthy control (HC), PTSD patients had lower smallworld index (Qi et al., 2017). However, other studies have revealed that the small-world properties of PTSD remain intact (Niu, Lei, et al., 2018). 
Although a few studies have examined the SCN in PTSD using graph theory, study on the topological characteristics of the structural brain network in PTSD remains limited. In addition, trauma-exposed control (TEC) and HC groups, which was essential to distinguish the pathological mechanism between PTSD and general trauma exposure, was not included and studied at the same time.

In our current research, the cortical thickness covariance brain networks of PTSD, TEC and HC groups was constructed. Comparison was thus made on the topological differences between the networks at the global and nodal level by using graph theory. Besides, the association between brain network characteristics and clinical scale in PTSD was explored. We hypothesized that PTSD and TEC groups have different degrees of variations on network indicators. It was also expected that alterations in the nodal properties of brain regions associated with stress and emotional processing. Prediction was made that the changes of SCN are closely related to the severity of clinical symptoms.

\section{Methods}

\section{Participants}

Recruitment for the research began in November 2014 and January 2015. A total of 102 subjects were recruited from a city named Wenchang in province of Hainan, China, which was severely hit by a tropical typhoon. On the basis of the Declaration of Helsinki, everyone submitted a written informed consent. At the same time, a protocol, which has been authorized by the ethics committee of Hainan General Hospital, were obtained for all subjects.

We only enrolled participants aged 18-65 years old with: right-handedness; no self-detailed history of head injury; no deficiency of awareness; no major clinical and neurological conditions; no comorbid lifetime; no current psychiatric disorder apart from anxiety and depression; no liquor and drug abuse/reliance; no utilization of mental medicine, or contraindications to MRI. At the time of study, none of the PTSD and TEC subjects were receiving any psychotherapy or medication. Eventually, 27 PTSD, 33 TEC and $29 \mathrm{HC}$ subjects were included in the research.

\section{MRI Data Acquisition}

All neuroimaging was conducted at the Hainan General Hospital using a Siemens 3T MR scanner with a 32-channel head coil. Head motion was reduced using foam pads and a Plexiglas head cradle. Structural MR images were collected for the whole brain with a sagittal 3D-magnetization prepared rapid gradient echo sequences. The parameters included TR/TE $=2300 / 1.97 \mathrm{~ms}$, field of view $=256 \mathrm{~mm}$, flip angle $\theta=$ $9^{\circ}$, matrix $=256 \times 256$, slice thickness $=1 \mathrm{~mm}, 176$ slices and scan duration $=353 \mathrm{~s}$. These scans were converted to NIFTI format for subsequent analysis.

\section{Clinical Measures}

The Clinician-Administered PTSD Scale (CAPS) (Weathers et al., 2001)was tested for evaluating the symptoms, supplemented by the PTSD Checklist-Civilian Version for DSM-IV (PCL-C) (Weathers et al., 
1993). Additional assessments to characterize the sample included the Self-Rating Anxiety Scale (SAS) for anxiety screening (Zung, 1971), the Self-Rating Depression Scale (SDS) for depression complications screening (Zung, 1965) and the Impact of Event Scale-Revised Scale (IES-R) (Weiss, 2007).

\section{Regional Cortical Thickness Measurement}

The reconstruction and estimation of the cerebral cortex of 68 cortical regions (bilateral; $34 \times 2$; DesikanKilliany Atlas) were obtained through an automatic processing stream called recon-all in FreeSurfer software package 6.0.0 (http://surfer.nmr.mgh.harvard.edu) (Dale et al., 1999; Fischl et al., 1999). A 10$\mathrm{mm}$ full-width at half-maximum Gaussian distribution was applied for image smoothing. Subsequently, all images were visually checked layer by layer and manually corrected when necessary.

\section{Construction of SCNs}

The Desikan-Killiany Atlas was utilized to divide the cerebral cortex. Pearson's correlation coefficients of cortical thickness between any node pairs were calculated in each group to form a matrix of $68 \times 68$. After excluding self-connections, negative weighted edges were set to zero (Gong et al., 2012; SanabriaDiaz et al., 2021). According to the predefined threshold sparsity ( $S=0.1: 0.01: 0.34$ ) (Zhang et al., 2011), the correlation coefficient greater than the threshold was retained, and the rest were set to 0 . Figure 1 showed the correlation matrix and binary matrix of each group.

\section{Network Attributes Calculation}

Different levels of properties for each threshold were calculated as followed: (1) Small-world topology: characteristic path length $\left(L_{p}\right)$, clustering coefficient $(C)$, small-world degree $(\sigma)$, normalized clustering coefficient $(\gamma)$ and normalized characteristic path length $(\lambda)$. The cluster coefficient reflects the degree of segregation of the network, and the characteristic path length indicates the extent of network integration. High clustering coefficient and short path length are the characteristics of small-world networks (Achard et al., 2006; Watts \& Strogatz, 1998). To evaluate the topological properties of brain networks, 100 null networks were generated and the average clustering coefficient $C_{\text {rand }}$ and the characteristic path length $L_{\text {rand }}$ were computed. Comparing the real network parameters $\left(C_{\text {rea }} \Omega L_{\text {real }}\right)$ with the random network parameters, $\gamma\left(\gamma=C_{\text {real }} / C_{\text {rand }}\right)$ and $\lambda\left(\lambda=L_{\text {real }} / L_{\text {rand }}\right)$ were obtained, and the $\sigma(\sigma=\gamma / \lambda)$ of the brain network was calculated. If $\sigma$ is significantly greater than 1, the brain network has a small world. (2) other whole brain network attributes: global efficiency $\left(E_{\text {global }}\right)$ and local efficiency $\left(E_{\text {local }}\right)$. (3) regional network attributes: nodal efficiency, nodal betweenness and nodal degree. These three indicators reflect the centrality of the node (Achard \& Bullmore, 2007; Freeman, 1977). Formulas and explanations for all these indicators could be found in previous literature (Bullmore \& Sporns, 2009). Each network index was calculated repeatedly within the selected density, and a graph was formed with the density as the abscissa and with a certain network parameter as the ordinate. This curve reflected the change of specific network indicators with network density. The area under the curve (AUC) of each network indicator was summarized for further analysis.

\section{Statistical Analysis}




\section{Demographic and Clinical Variables}

The independent sample t-tests, one-way analysis of variance and chi-square $\left(\chi^{2}\right)$ tests was performed according to the corresponding types of data ( $p \rrbracket .05)$. All the analyses were conducted in IBM Statistics SPSS 26, version 26.0 (IBM corp, Armonk, NY).

\section{Differences in network metrics}

The nonparametric permutation test was used to explore the difference in network topology between groups. Specifically, the difference in the mean value of a certain real measure between groups (for example, PTSD vs. TEC group) was first calculated, then all subjects were mixed and randomly assigned, and the correlation matrix of the randomized group was reconstructed. Based on these steps, the weight matrix was binarized and the difference of indicators between groups of the random network was calculated. 1000 permutations were repeated at each density to get a distribution of the difference. The $p$ value was based on the percentile of the real index difference $(p<.05)$. What's more, multiple comparisons between nodes were achieved using false discovery rate (FDR) correction $(p<.05)$. In all statistical analysis, the Brain Connectivity Toolbox (https://sites.google.com/ site/bctnet/) (Rubinov \& Sporns, 2010) was used in Matlab 2013b to construct and analyze the SCNs. The BrainNet Viewer (http://www.nitrc.org/projects/bnv/) was used to present changes in regional level.

\section{Relationship between Network Indicators and Clinical Variables}

Pearson correlation coefficients were calculated to evaluate the relationship between significant network measurement and clinical scale in patients with PTSD.

\section{Results}

\section{Cohort Characteristics}

27 patients with PTSD, 33 TEC and $29 \mathrm{HC}$ subjects were enrolled in this study (Table 1). No statistical differences in terms of gender distribution $\left(\chi^{2}=.190, d f=2, p=.909\right)$ and mean age $(F(2,86)=0.236, p$ $=.791)$ was revealed. The test for education level of three groups found significant differences $(F(2,86)=$ $8.255, p=.001)$, which was higher in HC group $(p<.005)$. Significant differences in terms of SAS $(F(2,86)$ $=80.015, p<.001)$ scores and SDS scores $(F(2,86)=99.425, p<.001)$ were also revealed. Further analyses showed that PTSD group had higher SAS and SDS scores (all $p<.001$ ). Among the PTSD patients, 9 subjects had depression comorbidities (seven females, two males), and one patient was diagnosed of anxiety disorder (female). The SDS scores of TEC group were significantly higher than those of HC group $(p<.05)$, while no significant difference as to SAS scores was found between these two groups $(p=0.088)$. The total scores of PCL $(t=13.738, d f=58, p<0.001)$ and subscale $\mathrm{B}(t=7.052$, $d f=58, p<.001)$, subscale $C(t=17.741, d f=58, p<.001)$ and subscale $D(t=10.131, d f=58, p<.001)$ in PTSD were found significantly higher than those in TEC subjects. 


\section{Network Analyses}

\section{Small-World Parameters}

\section{Global Network Measures}

The results of nonparametric replacement test showed no difference in the properties of global network between PTSD and HC subjects. The AUC analysis showed that the clustering coefficient $(p=.014)$ and local efficiency $\square=.031$ of PTSD group were higher than those of TEC group. The clustering coefficient $(p=.041)$ and local efficiency $(p=.027)$ of TEC group were significantly lower than those of HC group.

\section{Inter-Group Comparisons of Regional Network Measures}

An AUC analysis of the differences was performed in regional network indicators between the groups, however no nodes survived after FDR correction. Nevertheless, the inter-group comparison of node attributes still revealed the changes of some node characteristics of PTSD patients (Table 3 and Fig. 3). The main results are as followed: (1) PTSD vs. HC: In patients with PTSD, the nodal centralities of right isthmus cingulate cortex and right superior frontal gyrus increased. The nodal centralities of the left postcentral gyrus decreased. (2) PTSD vs. TEC: Compared with TEC group, the nodal centralities of PTSD on the left rostral middle frontal gyrus, bilateral supramarginal gyrus and right temporal pole increased significantly. The nodal degree of left insula of PTSD decreased. (3) TEC vs. HC: Compared with HC group, the nodal centralities of the left postcentral gyrus decreased, while the left insula increased significantly.

\section{Relationship between Network Indicators and Clinical Variables}

In PTSD group, after precluding the interferences of age, gender, education levels and ICV, partial correlation coefficients were computed between the significant brain network indicators and the clinical scales (Fig. 4). The result showed that there existed a significant positive correlation between clustering coefficient and CAPS score $(r=.706, p=.001)$. The increased nodal centralities were positively correlated with CAPS score $(r=.676, p=.002)$ in PTSD group.

\section{Discussion}

By using the method of graph theory analysis, the regional similarity of cortical thickness was calculated to ascertain the changes in topological characteristics of morphological networks of trauma-exposed patients. The relationship between these differences and clinical symptoms was also analyzed. As far as we know, this work is the first one to incorporate the structural covariance network of PTSD, TEC and HC 
groups simultaneously. We found that PTSD and TEC groups have different pathophysiological mechanisms, and the topological characteristics of brain network in both groups have changed significantly at different levels.

Human brain network is characterized by small-world attribute (Bullmore \& Sporns, 2009), which is associated with high-efficiency information dissemination with low construction cost (Achard \& Bullmore, 2007). The results of whole brain graph theory analysis showed that compared with HC group, PTSD patients retained the characteristics of small world topology. It is by now generally accepted that the small-world organization balances network isolation and network integration for information processing (Watts \& Strogatz, 1998). Other results have also reported the maintenance of small-world properties in PTSD group (Zhu et al., 2019). It was notable that the small-world attribute was found to be impaired in TEC group. This suggested that trauma could destroy the brain network topology attributes to a certain extent. Although trauma exposure in itself does not meet the diagnostic criteria, it still disrupts the balance of brain isolation and integration. However, it is still unclear whether the changes in small-world properties caused by trauma are temporary or permanent. A study of earthquake survivors found that within 25 days after the earthquake, the survivors' small-world attributes was found decreased compared with control group, indicating a shift towards randomized network, but the damage returned to normal after two years (Du et al., 2015).

Although the overall small-world structure of PTSD patients was similar to that of TEC subjects, their local efficiency and clustering coefficient were higher compared to TEC group, which was in line with previous studies (Lei et al., 2015; Proessl et al., 2020). The increase of clustering coefficient denotes higher fault tolerance of the brain when under attack from outside (Brust et al., 2012). In view of the inverse relationship between local efficiency and characteristic path length, the increase in local efficiency of PTSD may reflect more effective communication within network. The increase in local efficiency limits the transmission of information to closely connected specific areas, indicating the increase of network separation in the brains of PTSD patients. The changes of these global topology indexes were positively correlated with the severity of symptoms, indicating that the increased network separation has clinical significance.

The randomization process of neural networks is thought to be a common pattern in some neurological diseases (Lynall et al., 2010; Stam et al., 2009). In contrast, the increased local efficiency and clustering coefficient in current study indicated a more regular brain network in patients with PTSD. Regular patterns of brain networks have also been found in children with PTSD (Suo et al., 2015). While the networks of TEC subjects became increasingly random.

In addition to the indicators of the whole brain network, we also explored the changes at nodal level. The nodal efficiency of the right isthmus cingulate cortex of PTSD was higher than HC subjects. The alterations in the isthmus cingulate cortex reflect the symptoms of general neurological diseases, namely the impairments in emotional stimulation processing and response capability (Nielsen et al., 2005). Research has shown that PTSD patients have difficulty in emotional response (Wei et al., 2017). It is 
interesting to note that the nodal efficiency of the right isthmus cingulate cortex in patients with PTSD was closely related to the severity. Moreover, the nodal centrality in the right superior frontal gyrus of PTSD patients was greater than that of HC group. A similar pattern of results was obtained in previous studies (Sun et al., 2018; Suo et al., 2015). The superior frontal gyrus belongs to the prefrontal cortex and plays a critical role in the emotional brain circuit (Lee et al., 2004). In PTSD group, the higher nodal centrality of the right superior frontal gyrus may represent the neural changes related to emotional response in the brain. It is not clear whether these changes in nodal centrality reflect a pre-onset abnormality of PTSD or a consequence of trauma, since no such change was found in TEC group.

Compared with TEC subjects, PTSD patients demonstrated increased nodal centrality in the left rostral middle frontal gyrus, bilateral supramarginal gyrus and right temporal pole. The rostral middle frontal gyrus is the key brain area of emotion regulation, which is related to fear conditioning (Michalski et al., 2017; Minamoto et al., 2015). The supramarginal gyrus, which belongs to the parietal cortex, is related to emotional processing (Lee et al., 2004). The temporal pole is thought to be an extension of the limbic system (Giordana et al., 2005) that plays a key role in emotional processing (Ezzyat, 2007). In addition, it was also observed that these increased nodal centralities were positively correlated with CAPS scores, suggesting that these nodal lesions may be related to the severity of the disease.

The nodal centrality of the left postcentral gyrus in PTSD group and TEC group was lower than that of HC group, which suggested that this area was related to trauma. The postcentral gyrus is activated during the induction of basic negative emotions (Gilead et al., 2016; Ochsner et al., 2004). Previous studies found that the thickness of the postcentral gyrus decreased in PTSD patients (Lindemer et al., 2013; Liu et al., 2012). The nodal centrality in the left insula in the TEC group was higher than that of PTSD and HC group, suggesting that the left insula may be a protective factor against PTSD. Previous studies also found that the nodal degree of bilateral insula in trauma survivors increased significantly (Du et al., 2015). The insula is related to traumatic memory (King et al., 2009). The increase of nodal centrality of the left insula may improve the resistance of network topology to some extent, thereby reducing the damage of trauma. A previous study found that higher regional cerebral metabolic rate of glucose (rcMRglu) in the anterior insula was associated with stronger resilience, which suggest a role of rCMRgu in the anterior insula in resisting negative effect of trauma (Jeong et al.).

\section{Limitations}

There were some limitations in this study. Above all, the results of nodal attributes didn't survive correction, which might be due to sample constraints and limited capacity for statistical analysis. Expanding the sample size ought to be premeditated in future investigations. Secondly, the current research is only for typhoon victims, so it is necessary to be cautious in extending it to other types of trauma. Furthermore, as a cross-sectional study, it was uncertain whether the reported results existed preonset or were consequences of the diagnosis. In the future, longitudinal researches are expected to define the causal connection between brain network topology changes and PTSD. 


\section{Conclusions}

We compared the structural network topology among PTSD, TEC and HC groups based on graph theory. Our results confirmed that different levels of morphological network changes were associated with the severity of PTSD. At the global level, functional segregation of brain networks increased in PTSD patients, and brain networks tend to be regularized. In addition, at the node level, PTSD patients showed extensive changes in nodal centrality, involving the parietal lobe, frontal lobe and temporal lobe which were related to emotional processing. The brain networks of TEC patients tended to be randomized, and the increased nodal centrality of the left insula may be the protective factor of PTSD. Our study expanded the understanding of trauma-related pathobiology and evidenced the overlapping but distinct networks manifestations of PTSD and general trauma.

\section{Declarations}

Acknowledgments This study was supported by the National Natural Science Foundation of China (81871344囚81971602, 81760308); the Natural Science Foundation of Jiangsu Province (BK20191369); and the Qing Lan project of higher education institutions of Jiangsu Province.

Funding Not applicable.

Conflict of Interest The authors have no conflict of interest to declare.

Ethics approval According to the Declaration of Helsinki, all participants signed written informed consent. At the same time, a protocol, which has been approved by the ethics committee of Hainan General Hospital, were obtained for all subjects.

Consent to participate Informed consent was obtained from all patients for being included in the study.

Consent for publication All authors agree to publish this manuscript which has been read by all of them.

Availability of data and material Not applicable.

Code availability Not applicable.

Authors' contributions Author contributions included conception and study design ( $Y Z$ and GML), data acquisition (FC and LZ), data processing and statistical analysis (TTX, JK and RFQ), interpretation of results (TTX, FC and YZ), drafting the manuscript work or revising it critically for important intellectual content (TTX, FC, YLD and YZ) and approval of final version to be published and agreement to be accountable for the integrity and accuracy of all aspects of the work (All authors).

\section{References}


1. Achard, S., \& Bullmore, E. (2007). Efficiency and Cost of Economical Brain Functional Networks. PLOS Computational Biology, 3(2), e17-

2. Achard, S., Salvador, R., Whitcher, B., Suckling, J., \& Bullmore, E. (2006). A resilient, low-frequency, small-world human brain functional network with highly connected association cortical hubs. $J$ Neurosci, 26(1), 63-72. https://doi.org/10.1523/JNEUROSCI.3874-05.2006

3. Brust, M. R., Turgut, D., Ribeiro, C., \& Kaiser, M. (2012). Is the clustering coefficient a measure for fault tolerance in wireless sensor networks? Communications (ICC), 2012 IEEE International Conference on

4. Bullmore, E., \& Sporns, O. (2009). Complex brain networks: Graph theoretical analysis of structural and functional systems (Nature Reviews Neuroscience (2009) 10, (186-198)). Nature reviews Neuroscience, 10, 186-198.

5. Cai, S., Wang, X., Yang, F., Chen, D., \& Huang, L. (2021). Differences in Brain Structural Covariance Network Characteristics in Children and Adults With Autism Spectrum Disorder.Autism Research(1).

6. Dale, A. M., Fischl, B., \& Sereno, M. I. (1999). Cortical surface-based analysis. I. Segmentation and surface reconstruction. Neuroimage, 9(2), 179-194. https://doi.org/10.1006/nimg.1998.0395

7. Du, M. Y., Liao, W., Lui, S., Huang, X. Q., Li, F., Kuang, W. H. ... Gong, Q. Y. (2015). Altered functional connectivity in the brain default-mode network of earthquake survivors persists after 2 years despite recovery from anxiety symptoms. Soc Cogn Affect Neurosci, 10(11), 1497-1505. https://doi.org/10.1093/scan/nsv040

8. Ezzyat, P. Y. (2007). The Enigmatic temporal pole: a review of findings on social and emotional processing. Brain, 130(7), 1718-1731

9. Fischl, B., Sereno, M. I., \& Dale, A. M. (1999). Cortical surface-based analysis. II: Inflation, flattening, and a surface-based coordinate system. Neuroimage, 9(2), 195-207.

https://doi.org/10.1006/nimg.1998.0396

10. Francesmonneris, A., Pincus, H., \& First, M. (2013). Diagnostic and Statistical Manual of Mental Disorders: DSM-V. American Psychiatric Association

11. Freeman, L. C. (1977). A Set of Measures of Centrality Based on Betweenness. Sociometry, 40(1), 35-41

12. Gilead, M., Katzir, M., Eyal, T., \& Liberman, N. (2016). Neural correlates of processing "self-conscious" vs. "basic" emotions. Neuropsychologia,207-218.

13. Giordana, S., Sherwin, S. J., Peiró, J., Doorly, D. J., Papaharilaou, Y., Caro, C. G. ... Zervas, V. (2005). Automated classification of peripheral distal by-pass geometries reconstructed from medical data. $J$ Biomech, 38(1), 47-62. https://doi.org/10.1016/j.jbiomech.2004.03.015

14. Gong, G., Yong, H., Chen, Z. J., \& Evans, A. C. (2012). Convergence and divergence of thickness correlations with diffusion connections across the human cerebral cortex. Neuroimage, 59(2), 12391248

15. Guo, T., Guan, X., Zeng, Q., Xuan, M., Gu, Q., Huang, P. ... Zhang, M. (2018). Alterations of Brain Structural Network in Parkinson's Disease With and Without Rapid Eye Movement Sleep Behavior 
Disorder. Front Neurol, 9, 334. https://doi.org/10.3389/fneur.2018.00334

16. He, Y., Chen, Z. J., \& Evans, A. C. (2007). Small-world anatomical networks in the human brain revealed by cortical thickness from MRI. Cerebral Cortex(10),2407-2419.

17. Huang, Y., Wang, Y., \& Wang, H. (2019). Prevalence of mental disorders in China: a cross-sectional epidemiological study (vol 6, pg 211, 2019). Lancet Psychiatry, 6(4), E11-E11.

https://doi.org/10.1016/S2215-0366(19)30074-4

18. Jeong, H., Chung, Y. A., Ma, J., Kim, J., Hong, G., Jin, K. O. ... Yoon, S. Diverging roles of the anterior insula in trauma-exposed individuals vulnerable or resilient to posttraumatic stress disorder. Scientific Reports.

19. King, A. P., Abelson, J. L., Britton, J. C., Phan, K. L., Taylor, S. F., \& Liberzon, I. (2009). Medial prefrontal cortex and right insula activity predict plasma ACTH response to trauma recall. Neuroimage, 47(3), 872-880. https://doi.org/10.1016/j.neuroimage.2009.05.088

20. Lee, G. P., Meador, K. J., Loring, D. W., Allison, J. D., Brown, W. S., Paul, L. K. ... Lavin, T. B. (2004). Neural substrates of emotion as revealed by functional magnetic resonance imaging. Cogn Behav Neurol, 17(1), 9-17. https://doi.org/10.1097/00146965-200403000-00002

21. Lei, D., Li, K., Li, L., Chen, F., Huang, X., Lui, S. ... Gong, Q. (2015). Disrupted Functional Brain Connectome in Patients with Posttraumatic Stress Disorder. Radiology, 276(3), 818-827. https://doi.org/10.1148/radiol.15141700

22. Lindemer, E. R., Salat, D. H., Leritz, E. C., McGlinchey, R. E., \& Milberg, W. P. (2013). Reduced cortical thickness with increased lifetime burden of PTSD in OEF/OIF Veterans and the impact of comorbid TBI. Neuroimage Clin, 2, 601-611. https://doi.org/10.1016/j.nicl.2013.04.009

23. Liu, Y., Li, Y. J., Luo, E. P., Lu, H. B., \& Yin, H. (2012). Cortical thinning in patients with recent onset post-traumatic stress disorder after a single prolonged trauma exposure. PLoS One, 7(6), e39025. https://doi.org/10.1371/journal.pone.0039025

24. Lynall, M. E., Bassett, D. S., Kerwin, R., Mckenna, P. J., Kitzbichler, M., Muller, U., \& Bullmore, E. (2010). Functional Connectivity and Brain Networks in Schizophrenia. Journal of Neuroscience, 30(28), 9477-9487

25. Michalski, L. J., De Mers, C. H., Baranger, D., Barch, D. M., Harms, M. P., Burgess, G. C., \& Bogdan, R. (2017). Perceived stress is associated with increased rostral middle frontal gyrus cortical thickness: a family-based and discordant-sibling investigation. Genes Brain \& Behavior

26. Minamoto, T., Yaoi, K., Osaka, M., \& Osaka, N. (2015). The rostral prefrontal cortex underlies individual differences in working memory capacity: An approach from the hierarchical model of the cognitive control. Cortex.

27. Nielsen, F. A., Balslev, D., \& Hansen, L. K. (2005). Mining the posterior cingulate: segregation between memory and pain components. Neuroimage, 27(3), 520-532.

https://doi.org/10.1016/j.neuroimage.2005.04.034

28. Niu, R., Du, L., Chen, F., Chen, Y., Suo, X., Li, L. ... Gong, Q. (2018). Reduced local segregation of singlesubject gray matter networks in adult PTSD. Human Brain Mapping, 39. 
29. Niu, R., Lei, D., Chen, F., Chen, Y., Suo, X., Li, L. ... Gong, Q. (2018). Disrupted grey matter network morphology in pediatric posttraumatic stress disorder. Neuroimage Clin, 18, 943-951. https://doi.org/10.1016/j.nicl.2018.03.030

30. Ochsner, K. N., Ray, R. D., Cooper, J. C., Robertson, E. R., Chopra, S., Gabrieli, J. D., \& Gross, J. J. (2004). For better or for worse: neural systems supporting the cognitive down- and up-regulation of negative emotion. Neuroimage, 23(2), 483-499. https://doi.org/10.1016/j.neuroimage.2004.06.030

31. Proessl, F., Dretsch, M. N., Connaboy, C., Lovalekar, M., Dunn-Lewis, C., Canino, M. C. ... Flanagan, S. D. (2020). Structural Connectome Disruptions in Military Personnel with Mild Traumatic Brain Injury and Post-Traumatic Stress Disorder. J Neurotrauma, 37(19), 2102-2112. https://doi.org/10.1089/neu.2020.6999

32. Qi, S., Mu, Y. F., Cui, L. B., Zhang, J., Guo, F., Tan, Q. R. ... Zhang, N. Y. (2017). Anomalous gray matter structural networks in recent onset post-traumatic stress disorder. Brain Imaging \& Behavior

33. Rubinov, M., \& Sporns, O. (2010). Complex network measures of brain connectivity: uses and interpretations. Neuroimage, 52(3), 1059-1069

34. Sanabria-Diaz, G., Melie-Garcia, L., Draganski, B., Demonet, J. F., \& Kherif, F. (2021). Apolipoprotein E4 effects on topological brain network organization in mild cognitive impairment. Sci Rep, 11(1), 845. https://doi.org/10.1038/s41598-020-80909-7

35. Sporns, O., \& Zwi, J. D. (2004). The small world of the cerebral cortex. Neuroinformatics, 2(2), 145162

36. Stam, C. J., Haan, W. D., Daffertshofer, A., Jones, B. F., Manshanden, I., Van, C. ... Dijk, B. V. (2009). Graph theoretical analysis of magnetoencephalographic functional connectivity in Alzheimer's disease. Brain, 132(1), 213-224

37. Sun, D., Davis, S. L., Haswell, C. C., Swanson, C. A., Mid-Atlantic, M. W., LaBar, K. S. ... Morey, R. A. (2018). Brain Structural Covariance Network Topology in Remitted Posttraumatic Stress Disorder. Front Psychiatry, 9, 90. https://doi.org/10.3389/fpsyt.2018.00090

38. Suo, X., Lei, D., Li, K., Chen, F., Li, F., Li, L. ... Gong, Q. (2015). Disrupted brain network topology in pediatric posttraumatic stress disorder: A resting-state fMRI study. Hum Brain Mapp, 36(9), 36773686. https://doi.org/10.1002/hbm.22871

39. Suo, X., Lei, D., Li, W., Chen, F., Niu, R., Kuang, W. ... Gong, Q. (2019). Large-scale white matter network reorganization in posttraumatic stress disorder. Hum Brain Mapp, 40(16), 4801-4812. https://doi.org/10.1002/hbm.24738

40. Tijms, B. M., Seriès, P., Willshaw, D. J., \& Lawrie, S. M. (2012). Similarity-based extraction of individual networks from gray matter MRI scans. Cereb Cortex, 22(7), 1530-1541. https://doi.org/10.1093/cercor/bhr221

41. Watts, D. J., \& Strogatz, S. H. (1998). Collective dynamics of 'small-world' networks. Nature.

42. Weathers, F. W., Keane, T. M., \& Davidson, J. R. (2001). Clinician-administered PTSD scale: a review of the first ten years of research. Depress Anxiety, 13(3), 132-156. https://doi.org/10.1002/da.1029 
43. Weathers, F. W., Litz, B. T., Herman, D. S., Huska, J. A., \& Keane, T. M. (1993). The PTSD Checklist (PCL): Reliability, validity, and diagnostic utility.

44. Wei, C., Han, J., Zhang, Y., Hannak, W., \& Liu, Z. (2017). The Characteristics of Emotional Response of Post-traumatic Stress Disorder and Post-traumatic Growth among Chinese Adults Exposed to an Explosion Incident. Front Public Health, 5, 3. https://doi.org/10.3389/fpubh.2017.00003

45. Weiss, D. S. (2007). The Impact of Event Scale: Revised. In Wilson, J. P., \& Tang, C. S. (Eds.), CrossCultural Assessment of Psychological Trauma and PTSD (pp. 219-238). Springer US. https://doi.org/10.1007/978-0-387-70990-1_10

46. Zhang, J., Wang, J., Wu, Q., Kuang, W., Huang, X., He, Y., \& Gong, Q. (2011). Disrupted Brain Connectivity Networks in Drug-Naive, First-Episode Major Depressive Disorder. Biological Psychiatry, 70(4), 334-342

47. Zhang, J., Chen, Y. H., Rosa-Neto, P., Germann, J., \& Evans, A. C. (2008). Revealing Modular Architecture of Human Brain Structural Networks by Using Cortical Thickness from MRI. Cerebral Cortex, 18(10), 2374-2381

48. Zhu, H., Li, Y., Yuan, M., Ren, Z., Yuan, C., Meng, Y. ... Zhang, W. (2019). Increased functional segregation of brain network associated with symptomatology and sustained attention in chronic post-traumatic stress disorder. J Affect Disord, 247, 183-191.

https://doi.org/10.1016/j.jad.2019.01.012

49. Zung, W. W. (1965). A Self-Rating Depression Scale. Arch Gen Psychiatry, 12, 63-70. https://doi.org/10.1001/archpsyc.1965.01720310065008

50. Zung, W. W. (1971). A rating instrument for anxiety disorders. Psychosomatics, 12(6), 371-379. https://doi.org/10.1016/S0033-3182(71)71479-0

\section{Tables}

\section{Table 1}

The Demographic and Clinical Scores of 3 Groups 


\begin{tabular}{|c|c|c|c|c|}
\hline Characteris & PTSD $₫ n=27 \rrbracket$ & TEC $₫ n=33 \rrbracket$ & $\mathrm{HC} \bigotimes n=29 \rrbracket$ & $p$ \\
\hline \multicolumn{5}{|l|}{ Demographics } \\
\hline Gender & $7 / 20$ & $7 / 26$ & $7 / 22$ & $.909^{\mathrm{a}}$ \\
\hline \multicolumn{5}{|l|}{$\bigotimes$ males/females $\rrbracket$} \\
\hline Age $\bigotimes$ years $\rrbracket$ & $48.7 \pm 10.6$ & $48.6 \pm 7.5$ & $49.9 \pm 6.2$ & $.791^{\mathrm{b}}$ \\
\hline Education $\Downarrow$ years $\rrbracket$ & $6.4 \pm 3.4$ & $7.00 \pm 3.4$ & $9.8 \pm 3.3$ & $.001^{\mathrm{b}}$ \\
\hline \multicolumn{5}{|l|}{ Clinical data } \\
\hline SAS score & $52.6 \pm 10.6$ & $33.1 \pm 6.5$ & $28.8 \pm 4.4$ & $\otimes .001^{b}$ \\
\hline SDS score & $55.7 \pm 10.6$ & $33.0 \pm 7.3$ & $27.0 \pm 5.8$ & $\otimes .001^{b}$ \\
\hline PCL-B score & $17.2 \pm 4.0$ & $10.4 \pm 3.2$ & - & $\otimes .001^{c}$ \\
\hline PCL-C score & $19.5 \pm 3.0$ & $8.7 \pm 1.6$ & - & $\otimes .001^{c}$ \\
\hline PCL-D score & $17.1 \pm 3.0$ & $9.9 \pm 2.5$ & - & $\otimes .001^{c}$ \\
\hline PCL total score & $53.7 \pm 8.5$ & $28.9 \pm 5.4$ & - & $\otimes .001^{c}$ \\
\hline IES-B score & $20.7 \pm 4.6$ & - & - & - \\
\hline IES-C score & $16.9 \pm 4.5$ & - & - & - \\
\hline IES-D score & $15.5 \pm 3.9$ & - & - & - \\
\hline IES total score & $52.9 \pm 11.2$ & - & - & - \\
\hline CAPS-B score & $24.5 \pm 7.3$ & - & - & - \\
\hline CAPS-C score & $28.1 \pm 8.3$ & - & - & - \\
\hline CAPS-D score & $25.6 \pm 6.8$ & - & - & - \\
\hline CAPS total score & $78.2 \pm 19.3$ & - & - & - \\
\hline \multicolumn{5}{|c|}{$\begin{array}{l}\text { Data are shown as mean } \pm \text { SD except gender, which is presented by the number of each option. PTSD } \\
\text { post-traumatic stress disorder; TEC, trauma-exposed control; HC, healthy control; SAS, Self-Rating } \\
\text { Anxiety Scale; SDS, Self-Rating Depression Scale; PCL, PTSD Checklist; IES, Impact of Event Scale; } \\
\text { CAPS, Clinician-Administered PTSD Scale. }\end{array}$} \\
\hline \multicolumn{5}{|c|}{ a $p$ value got using a chi-square test. } \\
\hline \multicolumn{5}{|c|}{${ }^{\mathrm{b}} p$ value got with one-way analysis of variance. } \\
\hline
\end{tabular}




\section{Table 2}

Global Network Properties between Groups

\begin{tabular}{|llll|}
\hline & PTSD vs. HC & PTSD vs. TEC & TEC vs. HC \\
\hline$C$ & .816 & $.014^{*}$ & $.041^{*}$ \\
\hline$L_{p}$ & .928 & .285 & .372 \\
\hline$E_{\text {global }}$ & .717 & .243 & .433 \\
\hline$E_{\text {local }}$ & .949 & $.031^{*}$ & $.027^{*}$ \\
$\gamma$ & .842 & .227 & .141 \\
\hline$\lambda$ & .947 & .550 & .474 \\
\hline$\sigma$ & .877 & .304 & $.017 *$ \\
\hline * represented the $p<.05 . C$, clustering coefficient; $L_{p,}$ character path length; $E_{\text {global, }}$ global efficiency; \\
$\begin{array}{l}E_{\text {local }} \text { local efficiency; } \gamma, \text { normalized clustering coefficient; } \lambda \text {, normalized characteristic path length; } \sigma \\
\text { small-world index. PTSD, post-traumatic stress disorder; } \text { TEC, trauma-exposed control; HC, healthy } \\
\text { control. }\end{array}$
\end{tabular}

\section{Table 3}

$P$-value for Regions Exhibiting Significant Between-group Differences in Nodal Centralities. 


\begin{tabular}{|llll|}
\hline & P value & & \\
\hline Group comparison & Nodal efficiency & Nodal betweenness & Nodal degree \\
\hline PTSD $>$ HC & & & \\
\hline Rh isthmus cingulate & $.042^{\star}$ & .089 & .092 \\
\hline Rh superior frontal & .257 & $.044^{\star}$ & .302 \\
\hline PTSD $<$ HC & & & $.018^{\star}$ \\
\hline Lh postcentral & $.028^{\star}$ & $.012^{\star}$ & $.037^{\star}$ \\
\hline PTSD $>$ TEC & & $.032^{\star}$ & $.044^{\star}$ \\
\hline Lh rostral middle frontal & $.025^{\star}$ & .179 & .367 \\
\hline Lh supramarginal & $.015^{\star}$ & .195 & .054 \\
\hline Rh supramarginal & $.034^{\star}$ & .203 & $.023^{\star}$ \\
\hline Rh temporal pole & $.011^{\star}$ & & $.027^{\star}$ \\
\hline PTSD $<$ TEC & & .268 & \\
\hline Lh_insula & .059 & $.032^{\star}$ & \\
\hline TEC > HC & & $.01^{\star}$ & \\
\hline Lh insula & & & \\
\hline TEC < HC & & & \\
\hline Lh postcentral & & & \\
\hline
\end{tabular}

Regions were regarded as abnormal if they displayed significant between-group differences in at least one of the three nodal centralities. ${ }^{*} p<.05$, uncorrected. ${ }^{*} p<.01$, uncorrected. Lh, left hemisphere; Rh, right hemisphere; PTSD, post-traumatic stress disorder; TEC, trauma-exposed control; $\mathrm{HC}$, healthy control.

\section{Figures}


Correlation matrix of PTSD

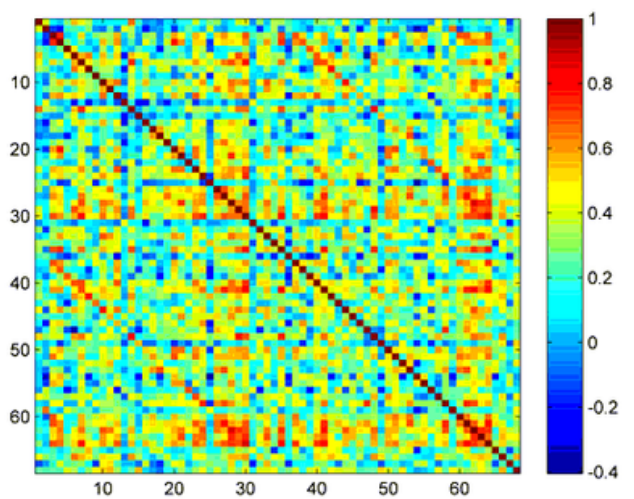

Binary graph of PTSD

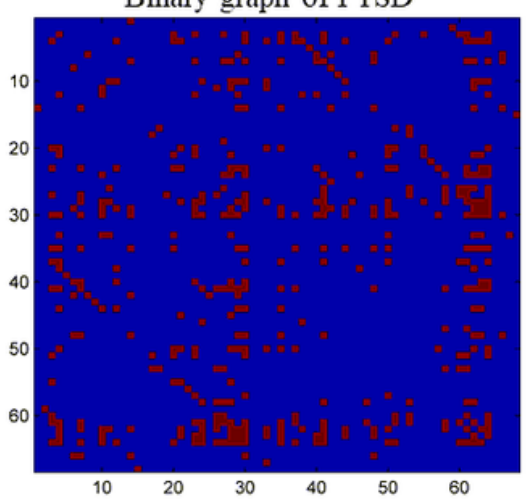

Correlation matrix of TEC

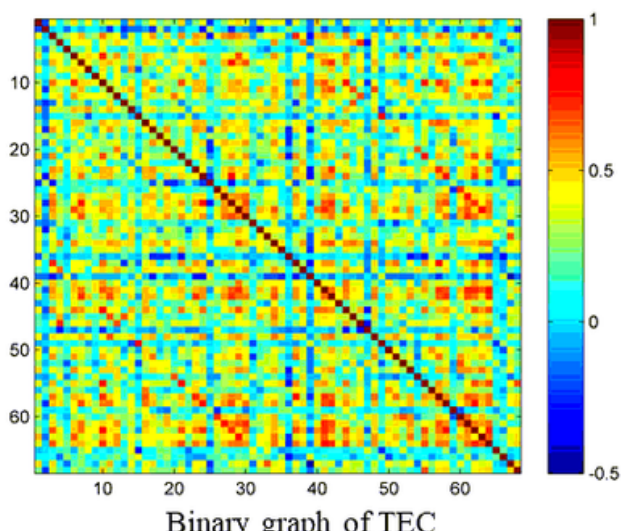

Binary graph of TEC

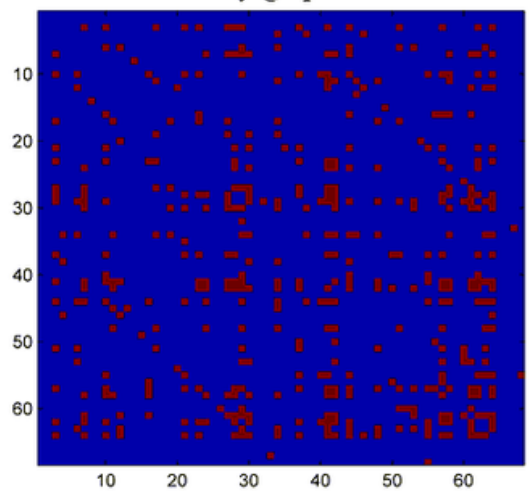

Correlation matrix of $\mathrm{HC}$

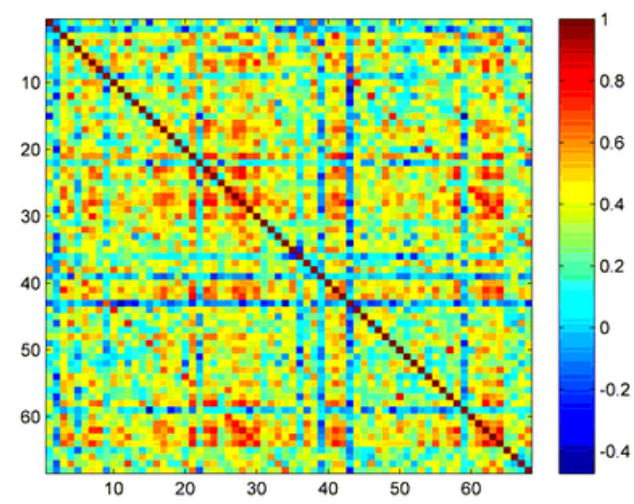

Binary graph of $\mathrm{HC}$

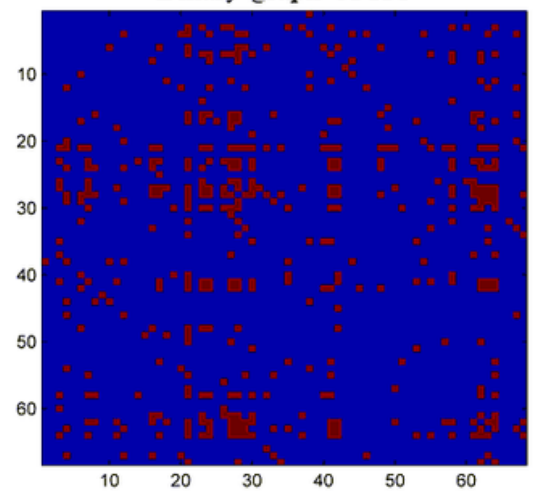

\section{Figure 1}

Correlation matrix of each group of cortex thickness. The upper row is the cortical thickness correlation matrices of PTSD $\square T E C \square H C$ groups after excluding self-connections. The color bar indicates the strength of the correlation coefficients. The lower row is the binary matrix for each group. PTSD, post-traumatic stress disorder; TEC, trauma-exposed control; $\mathrm{HC}$, healthy control. 
A

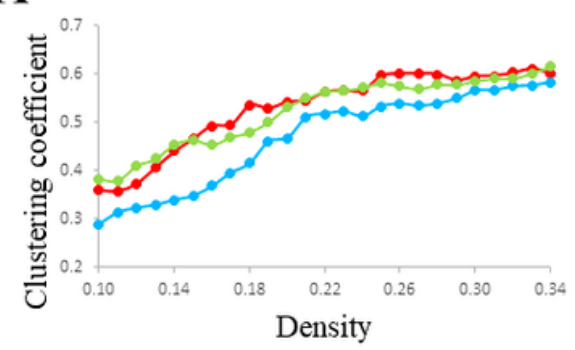

C

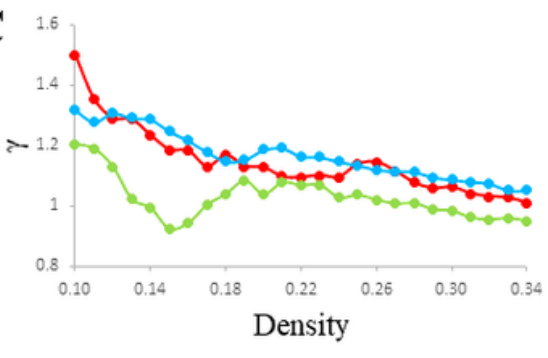

B

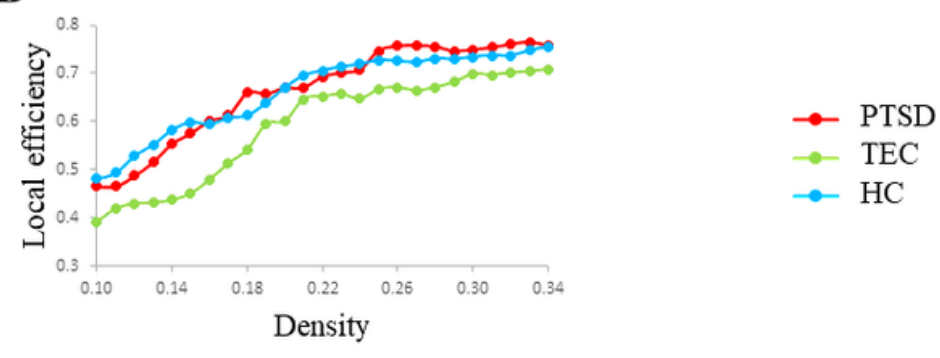

D

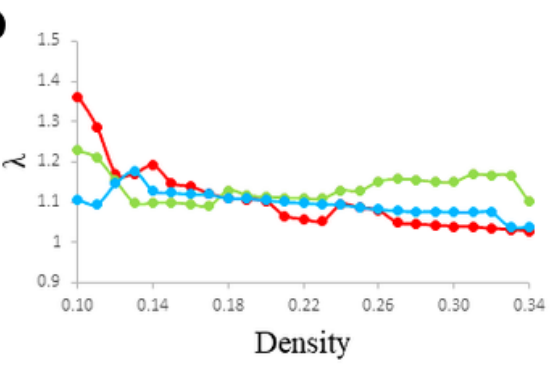

$\mathbf{E}$

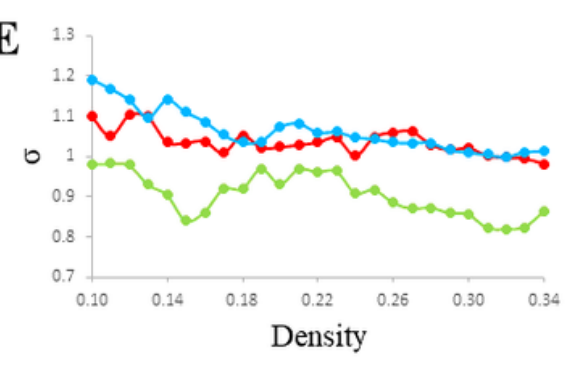

\section{Figure 2}

Significant global network measures of the $S C N$ as a function of network density (0.1:0.01:0.34). Individual graphs show the (A) clustering coefficient, (B) local efficiency, (C) $\gamma$, normalized clustering coefficient, (D) $\lambda$, normalized characteristic path length, (E) $\sigma$, small-world index. PTSD, post-traumatic stress disorder; TEC, trauma-exposed control; $\mathrm{HC}$, healthy control. 


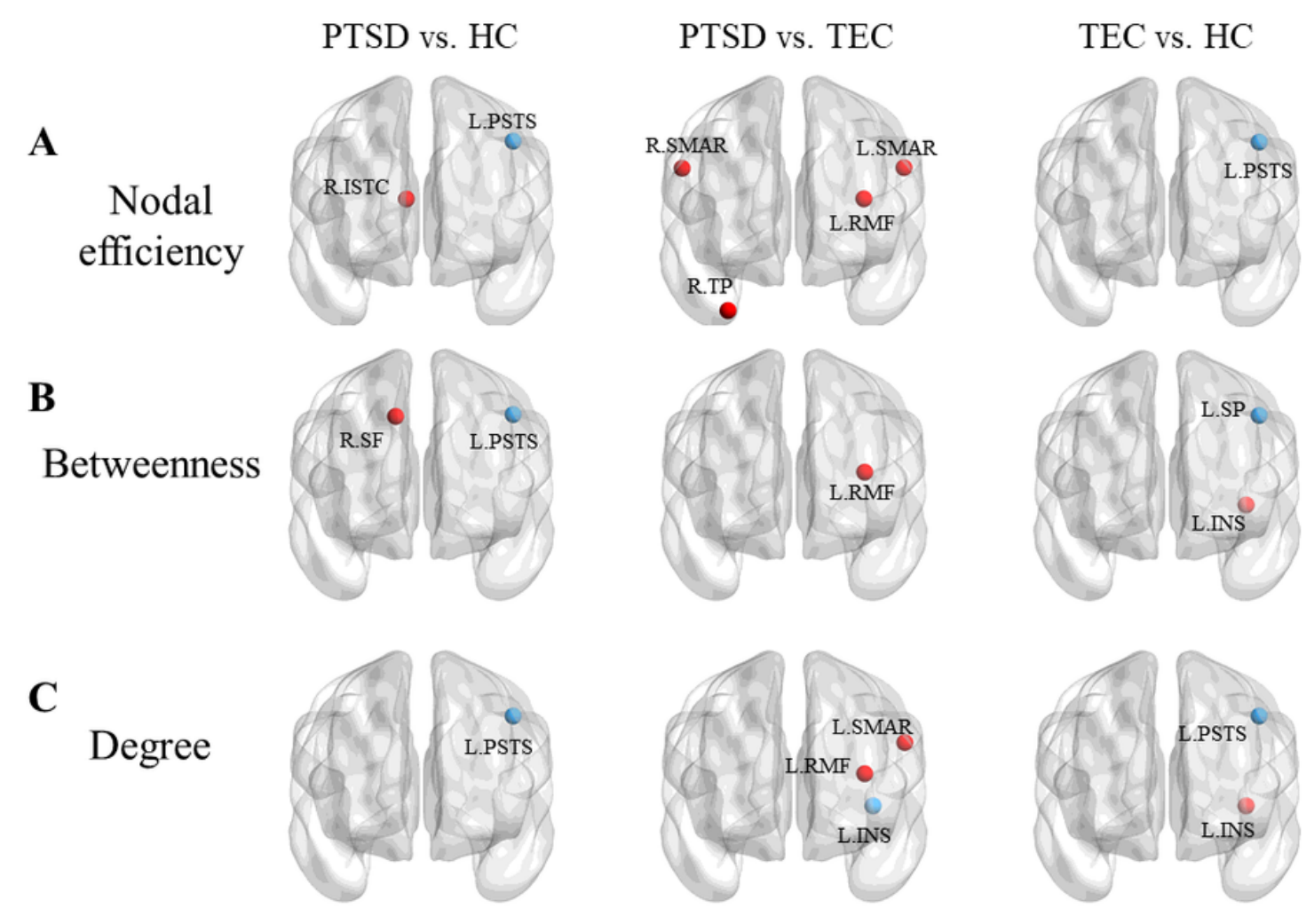

Figure 3

Regions showing significant between-group differences in nodal centralities $(p<.05$, uncorrected). Subgraphs show differences for the (A)nodal efficiency, (B)betweenness, (C)degree. Red nodes indicate increased nodal centralities in front group, blue nodes indicate decreased nodal centralities in front groups. L, left; R, right; ISTC, isthmus cingulate cortex; SF, superior frontal gyrus; PSTS, postcentral gyrus; RMF, rostral middle frontal gyrus; SMAR, supramarginal gyrus; TP, temporal pole; INS, insula; PTSD, posttraumatic stress disorder; TEC, trauma-exposed control; $\mathrm{HC}$, healthy control. 

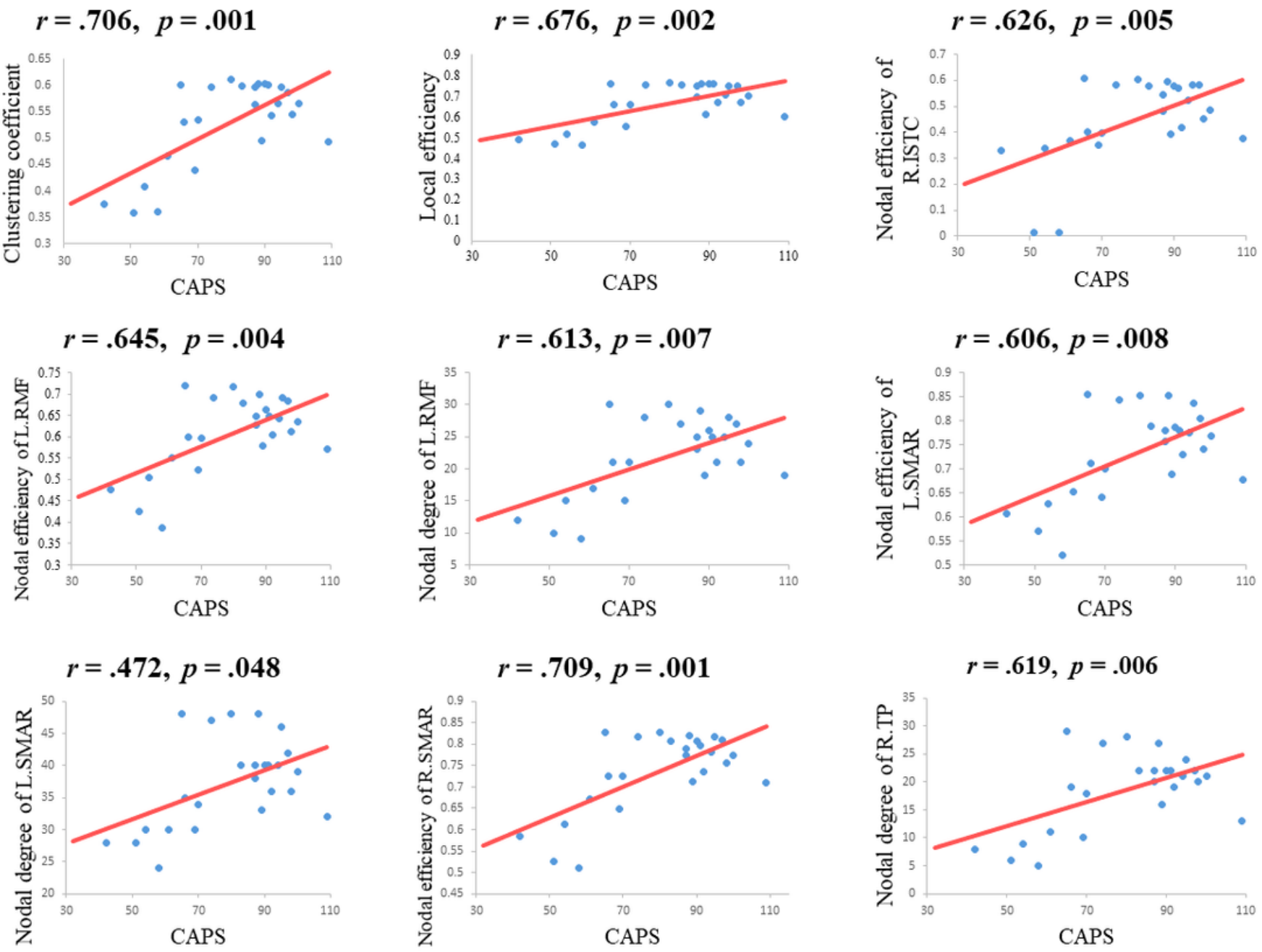

\section{Figure 4}

Scatter plots of global and nodal measures against CAPS scores in PTSD with a significant correlation ( $p$ $<.05)$. L, left; R, right; ISTC, isthmus cingulate cortex; RMF, rostral middle frontal gyrus; SMAR, supramarginal gyrus; TP, temporal pole. 